\title{
Driving trajectories in chaotic scattering
}

\author{
Elbert E. N. Macau* \\ Laboratório de Integraçāo e Testes (LIT), Instituto Nacional de Pesquisas Espaciais (INPE), São José das Campos, São Paulo, Brazil \\ Iberê L. Caldas ${ }^{\dagger}$ \\ Departamento de Física Aplicada, Universidade do Estado de São Paulo (USP), Instituto de Física (IF), 05315-97 São Paulo, Brazil
}

(Received 22 June 2001; published 24 January 2002)

\begin{abstract}
In this work we introduce a general approach for targeting in chaotic scattering that can be used to find a transfer trajectory between any two points located inside the scattering region. We show that this method can be used in association with a control of chaos strategy to drive around and keep a particle inside the scattering region. As an illustration of how powerful this approach is, we use it in a case of practical interest in celestial mechanics in which it is desired to control the evolution of two satellites that evolve around a large central body.
\end{abstract}

DOI: 10.1103/PhysRevE.65.026215

PACS number(s): 05.45.- a

\section{INTRODUCTION}

The phenomenon of chaotic scattering has been identified in many fundamental physical situations, such as celestial mechanics [1], molecular dynamics [2], fluid dynamics [3,4], mesoscopic systems [5], chemical reactions [6,7], electrodynamics [8], and nuclear physics [9]. The term scattering characterizes a situation that may happen in open systems. Depending on specific control parameters, the motion can be regular or chaotic in the scattering region. A scattering is usually described by the scattering function, which relates to the final state of a scattered particle, a function of its initial state. In a chaotic scattering, the scattering function presents a cantor set of singularities [10]. This set of singularities is due to the existence of a chaotic nonattractive invariant set in the scattering region. Thus, initial conditions on this cantor set of singularities of the scattering function imply in trajectories that enter the scattering region, stay wandering the chaotic set and so never exit. Furthermore, in the neighborhood of those initial conditions, the system presents extremely high sensitive dependence on small perturbations, which is the hallmark of chaos.

In this work we show that this sensitive dependence on small perturbations can be used not only to keep a particle that comes from the outside in the scattering region, but also to change its trajectory so that it describes an elaborated desired motion inside the scattering region. This capability is usefull in practical situations, as are the cases of chemical reactions, particle accelerators, electromagnetic wave generators, among others, where the efficiency of the respective processes could be enhanced if the system could be guided to specific trajectories in its state space. In fact, in Ref. [11], the authors applied a method inspired on the Ott-Grebogi-Yorke (OGY) method of control of chaos to stabilize trajectories that come from outside the scattering region on one of the unstable periodic orbits embedded in the chaotic set. However, the authors stressed that the major problem about con-

\footnotetext{
*Email address: elbert@lit.inpe.br

${ }^{\dagger}$ Email address: ibere@if.usp.br
}

trol of chaotic scattering is to bring the trajectories inside the region where their method could be applied ("controllable region"). This is because the chaotic set in a chaotic scattering is nonattractive, so there is just a very small probability that a trajectory that starts from a random chosen initial condition enters the "controllable" region. In Ref. [12], the author introduced a targeting method that uses small perturbations to drive trajectories from an unstable periodic orbit located inside the chaotic scattering region to a target point outside the scattering region. Here, we generalize this method so that it can be used to quickly transfer between any two points located inside the chaotic scattering. Furthermore, we show that when the trajectory is about an unstable periodic orbit, the formerly described control of chaos method can be applied to keep the trajectory evolving on the unstable periodic orbit. Thus, the combination of chaos control with targeting gives us a powerful approach that can be used to direct a trajectory inside a chaotic scattering to produce elaborated trajectories.

To demonstrate our approach, we consider the situation that appears in Fig. 1, where two light bodies $M_{2}$ and $M_{3}$ describe initially coplanar and circular orbits, with slightly different radii, around a heavy central body $M_{1}$. In this scenario, our goal is to use our control of chaos strategy to control the orbital evolution of $M_{2}$ and $M_{3}$. If the two light bodies are far apart, their mutual attraction can be neglected, and the problem reduces in a fair approximation to a superposition of two independent two-body problems. However, if the distance between them becomes sufficiently small, a situation called as an encounter, their mutual attraction is no longer negligible and cannot be ignored. This is known as Hill's problem [13].

The Hill's problem is known as the simplest nonintegrable case of the $N$-body problem. The equations are very simple, and contain no parameter. However, many problems in celestial mechanics can be adequately approximated by Hill's equations. Examples are the Sun-Earth-Moon problem [13], which was the motivation of Hill's original work; the interaction between particles in planetary rings [14-16]; the motion of coorbital satellites [17]; the accretion of particles by a 


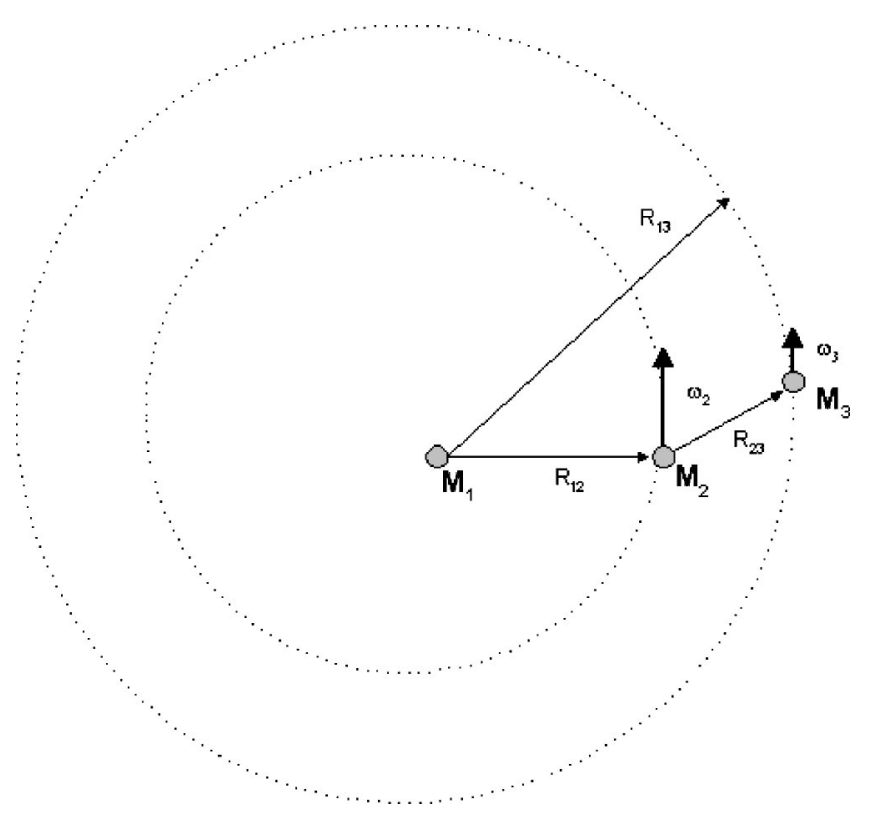

FIG. 1. Schematic representation of the problem. Two light bodies describing initially coplanar and circular orbits, with slightly different radii, around a heavy central body. In the initial situation $M_{2}$, the inner body, has a slightly larger angular velocity than $M_{3}$, which means that after some time they eventually interact gravitationally with each other.

protoplanet [18]; and the distribution of particles around the Earth [19].

In the present paper we use the Hill's problem to model the case where $M_{2}$ and $M_{3}$ are artificial satellites or spacecrafts, and our goal is to stabilize the satellites in an orbit in which they evolve around each other. The difference between the radii of their initial circular orbits is called the impact parameter. In the initial situation; $M_{2}$, the inner body, has a slightly larger angular velocity than $M_{3}$, which means that after some time they eventually go to an encounter. Depending on the impact parameter, the result of the encounter is (i) only a slight deflection on their previous circular orbits, if the impact parameter is sufficiently large; (ii) a "horseshoe" motion, in which the bodies "repel" each other azimuthally and never come in close proximity, if the impact parameter is very small; (iii) a very evolved and complex motion characterized by "sensitive dependence" of the motion after the encounter to small changes in the impact parameter and by singularities in the scattering function. This last situation is in fact a case of a chaotic scattering $[20,12]$. Our strategy is used in this region of the impact parameter where a chaotic scattering appears to exploit advantageously the characteristics of a chaotic motion to guide the movement of the artificial satellites.

This paper is organized as follows. In Sec. II we review the basic theory of the OGY method and present a formulation for parameter perturbations that does not explicitly involve eigenvalues. In Sec. III we present our targeting method in a situation of a chaotic scattering. In Sec. IV we derive the Hill's equations and we analyze the dynamical behavior of the Hill's equation and its correlation with a chaotic scattering scenario. In Sec. V we apply our methods to get the desired satellite control behavior. In Sec. VI we present conclusions and general discussions.

\section{STABILIZING CHAOTIC TRAJECTORIES}

One fundamental aspect of chaotic invariant sets is that they are typically permeated by an infinite dense set of unstable periodic orbits. Control of chaos by stabilizing those unstable periodic orbits was conceived by Ott, Grebogi, and Yorke $[20,21]$. Their basic ideas regarding a chaotic attractor is as follows. First one chooses an unstable periodic orbit embedded in the chaotic set according to some performance criterion. Second, one defines a small region around the desired periodic orbit. As a chaotic trajectory is transitive in its invariant set, starting from any initial condition, after some time it will come to that small region about the chosen unstable periodic orbit. When this occurs, small judiciously chosen parameter perturbations are applied to force and keep the trajectory evolving on the unstable periodic orbit. In subsequent works, Lai, Tel, and Grebogi [22,11] showed that with small modifications the OGY method can be applied to hyperbolic Hamiltonian systems, as follows.

Let us consider the above discrete time dynamical system,

$$
\mathbf{X}_{i+1}=\mathbf{F}\left(\mathbf{X}_{i}, p\right),
$$

where $\mathbf{X}_{i} \in \mathfrak{R}^{2}, p \in \mathfrak{R}$ is an externally controllable parameter, and $\mathbf{F}$ is a smooth function in both variables. The parameter perturbation to be used to control the system is required to be small, i.e., it is required that

$$
\left|p-p_{0}\right|<\delta
$$

where $p_{0}$ is some nominal parameter value and $\delta$ is a small number defining the range of parameter variation. The goal is to program the parameter $p$ in such a way that a typical trajectory in the chaotic region is stabilized about the desirable unstable periodic orbit. The stabilization procedure just starts to actuate when the chaotic trajectory enters a small region around one of the periodic-orbit point, whose size is proportional to $\delta$. Once the particle is inside this small region, $p$ is judiciously changed to keep the trajectory around the unstable periodic orbit.

Let us assume the unstable periodic orbit (UPO) of period $m$ to be controlled is

$$
\mathbf{X}_{01}(p) \rightarrow \mathbf{X}_{02}(p) \cdots \rightarrow \mathbf{X}_{0 m}(p) \rightarrow \mathbf{X}_{0(m+1)}(p)=\mathbf{X}_{01}(p)
$$

The linearized dynamics in the neighborhood of the period- $m$ orbit is

$$
\mathbf{X}_{n+1}-\mathbf{X}_{0(n+1)}\left(p_{n}\right)=\mathbf{M}\left[\mathbf{X}_{n}-\mathbf{X}_{0 n}\left(p_{n}\right)\right],
$$

where $\mathbf{M}$ is the two-dimensional Jacobian matrix at the orbit point $\mathbf{X}_{0 n}, p_{n}=p_{0}+(\Delta p)_{n} \mathbf{g}_{n}$ where from Eq. (2) $(\Delta p)_{n}$ $<\delta$. Observe that the parameter variation will result in the following change in the periodic-orbit points:

$$
\mathbf{X}_{0 n}\left(p_{n}\right)-\mathbf{X}_{0 n}\left(p_{0}\right) \approx(\Delta p)_{n} \mathbf{g}_{n},
$$

where $g_{n}=\partial \mathbf{X}_{0 n}(p) /\left.\partial p\right|_{p_{0}}$. 


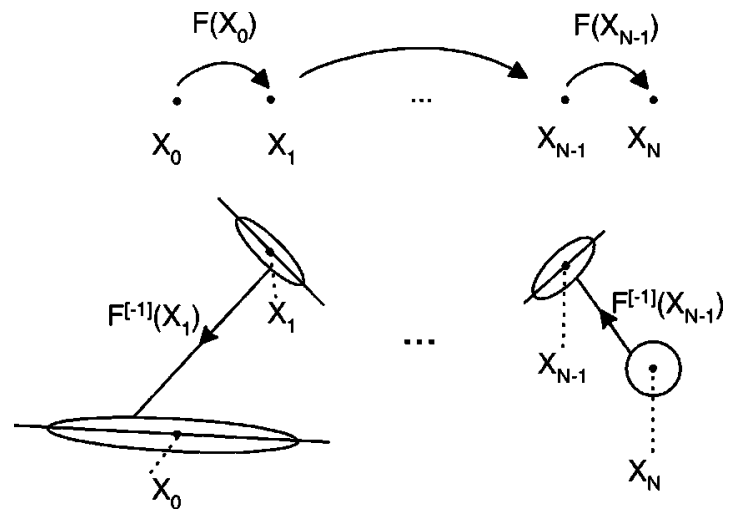

FIG. 2. Schematic illustration of the procedure used to find the stable direction at a point $X_{0}$ : A circle of arbitrarily small radius is put at the point $X_{n}$ and is iterated backward $N$ times. The resulting ellipse has its major axis along the stable direction of $X_{0}$, provided that $N$ is large enough.

The Jacobian matrix $\mathbf{M}$ of Eq. (4) can be expressed in terms of its stable and unstable directions. Note that even in the case in which $\mathbf{M}$ has complex-conjugate eigenvalues, the stable and unstable directions for $\mathbf{M}$ can be defined. To find the stable direction at a point $\mathbf{X}_{0}$, we first iterate this point forward $N$ times under the map $\mathbf{F}$ and get the trajectory $\mathbf{X}_{1}$ $=\mathbf{F}\left(\mathbf{X}_{0}\right), \quad \mathbf{X}_{2}=\mathbf{F}\left(\mathbf{X}_{1}\right)=\mathbf{F}^{|2|}\left(\mathbf{X}_{0}\right), \ldots, \quad$ and $\quad \mathbf{X}_{N}=\mathbf{F}\left(\mathbf{X}_{N-1}\right)$ $=\mathbf{F}^{|N|}\left(\mathbf{X}_{0}\right)$. Now, a circle of arbitrarily small radius $\epsilon$ is put at the point $\mathbf{X}_{N}$. If this circle is iterated backward once, the circle will become an ellipse at the point $\mathbf{X}_{N-1}$ with the major axis along the stable direction of the point $\mathbf{X}_{N-1}$. This ellipse is iterated backward, while at the same time its major axis is kept of order $\epsilon$ via certain normalization method. This procedure is repeated all the way back to the point $\mathbf{X}_{0}$, where the ellipse becomes very thin, with its major axis along the stable direction provided $N$ is large enough. This procedure is schematically shown in Fig. 2.

Similarly, as shown in Fig. 3, to find the unstable direction at point $\mathbf{X}_{0}$, first this point is iterated backward under the inverse map $N$ times to get a backward orbit $\mathbf{X}_{-j}$ $=\mathbf{F}^{|-j|}\left(\mathbf{X}_{0}\right)(j=1, \ldots, N)$. In the point $\mathbf{X}_{-N}$ a circle of arbi-
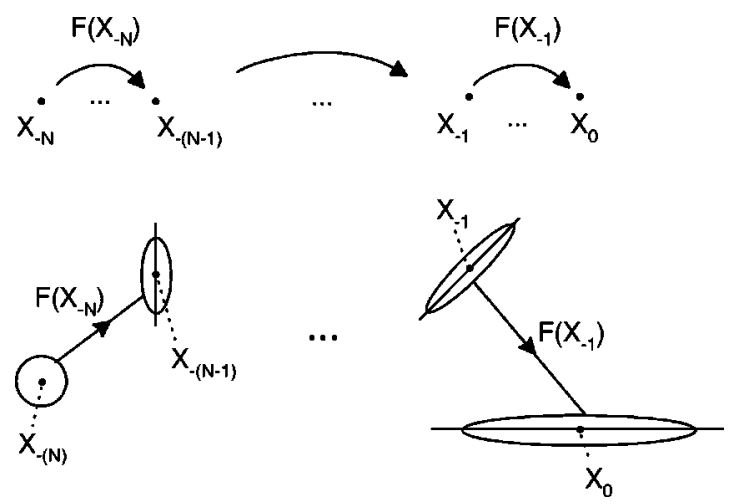

FIG. 3. Schematic illustration of the procedure used to find the unstable direction at a point $X_{0}$ : A circle of arbitrarily small radius is put at the point $X_{-n}$ and is iterated forward $N$ times. The resulting ellipse has its major axis along the unstable direction of $X_{0}$, provided that $N$ is large enough. trarily small radius $\epsilon$ is put at it. After that, the previously discussed procedure is applied, but now iterating in the forward direction. At the end of this procedure, in $\mathbf{X}_{0}$ the ellipse becomes very thin, with its major axis along the unstable direction.

Let $\mathbf{e}_{s(n)}$ and $\mathbf{e}_{u(n)}$ be the stable and unstable directions at $\mathbf{X}_{0 n}$, and let $\mathbf{f}_{s(n)}$ and $\mathbf{f}_{u(n)}$ be two vectors that satisfy $\mathbf{f}_{u(n)} \cdot \mathbf{e}_{u(n)}=\mathbf{f}_{s(n)} \cdot \mathbf{e}_{s(n)}=1$ and $\mathbf{f}_{u(n)} \cdot \mathbf{e}_{s(n)}=\mathbf{f}_{s(n)} \cdot \mathbf{e}_{u(n)}=0$. To control the orbit, it is required that the next iteration of a trajectory point after falling into one of the small neighborhoods around $\mathbf{X}_{0 n}$ lies on the stable direction at $\mathbf{X}_{0(n+1)}$ $\times\left(p_{0}\right)$, i.e.,

$$
\left[\mathbf{X}_{n+1}-\mathbf{X}_{0(n+1)}\left(p_{0}\right)\right] \cdot \mathbf{f}_{u(n+1)}=0 .
$$

Substituting Eqs. (4) and (5) into Eq. (6), we obtain the following expression for the parameter perturbations:

$$
(\Delta p)_{n}=\frac{\left\{\mathbf{M}\left[\mathbf{X}_{n+1}-\mathbf{X}_{0(n+1)}\left(p_{0}\right)\right]\right\} \cdot \mathbf{f}_{u(n+1)}}{\left[\left(\mathbf{M g}_{n}\right)-\mathbf{g}_{n+1}\right] \cdot \mathbf{f}_{u(n+1)}},
$$

where $\mathbf{M}$ is evaluated at $\mathbf{X}_{0 n}\left(p_{0}\right)$. This parameter perturbation is applied at each time step of the trajectory, which is so kept stabilized around the unstable periodic orbit.

\section{TARGETING IN CHAOTIC SCATTERING}

The inherent exponential sensitivity of chaotic time evolutions to perturbations is the hallmark of chaotic systems. This characteristic is responsible for the impossibility of making long-term predictions of the system evolution based on finite precision measurement. However, despite the complexities of chaotic behavior, this same main characteristic can be intelligently exploited to direct a system to some desired state using a carefully chosen sequence of small perturbations to some system parameter. This approach was introduced by Shinbrot, Ott, Grebogi, and Yorke [23] and have been called targeting. In the context of chaotic scattering, Ref. [12] proposed an approach applicable to the situation where it is desirable to target some region of phase space out of scattering region to a particular point $P$ from an unstable periodic orbit $C_{u}$ located inside the scattering region. Thus, by applying small perturbing control, the goal was to direct the motion of the particle so that it hits the point $P$. The author assumed that initially the unstable periodic orbit is kept stabilized with the use of a control of chaos strategy. In what follows, we introduce substantial changes in this method to make it applicable to transfer between any two points located inside the chaotic scattering region.

If our system is a continuous time system (flow), we can introduce a convenient surface of section transverse to the flow so that the study of the continuous time system is reduced to the study of an associated discrete time system, the Poincaré map. We will denote by $f$ this map that transforms the point $\left(s_{n}\right)$, located in the surface of section, to the point $\left(s_{n+1}\right)$, associated with the first return of the flow to the surface of section, i.e., $f:\left(s_{n}\right) \rightarrow\left(s_{n+1}\right)$.

Let us suppose that our goal is to target a point $P_{g}$ from a point $Q_{g}$ located inside the scattering region. Starting from $Q_{g}$, we find the first point $Q$ in which the trajectory crosses 


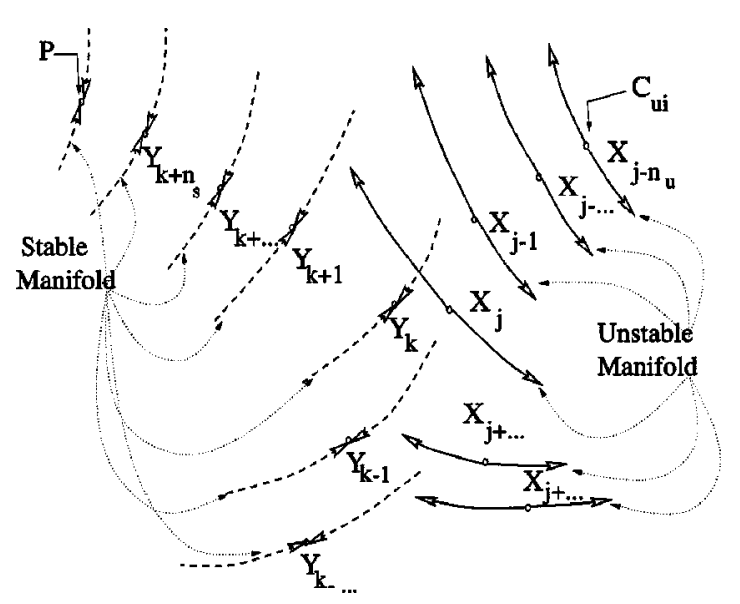

FIG. 4. Two trajectories that come close to each other.

the surface of section. We repeat this procedure, this time starting from the point $P_{g}$ to find the point $P$ also located on the surface of section. Now, our aim is to find a "small" perturbation $\delta s$ so that if those numbers are adequately applied to the original trajectory that passes through $Q$ we have a perturbed trajectory that eventually hits a target point $P$, which is also located on the surface of section.

Starting at $Q$ we randomly choose a large number of nearby initial conditions. We choose an integer number $L$ and retain those initial conditions $\left\{r_{0}^{1}, r_{0}^{2}, \ldots, r_{0}^{j}, \ldots\right\}$ that lead to a set of trajectories $\left\{r^{1}, \ldots, r^{j}, \ldots\right\}$ that remain on the scattering region for at least $2 \times L$ crossing times the surface of section. For $L$ large enough, these initial conditions are located near the stable manifold of the chaotic invariant set [24]. Furthermore, for each trajectory its middle point $\left(r_{\mathrm{md}}^{j}\right)$ and its antecessor $\left(r_{\mathrm{md}-1}^{j}\right)$ and succeeder $\left(r_{\mathrm{md}+1}^{j}\right)$ points are located very close to the chaotic invariant set [24]. Let us call $I_{Q}$ the set of those points. Starting at $P$ we randomly choose a large number of nearby initial conditions. We set a number $N$ and retain those initial conditions $\left\{s_{0}^{1}, s_{0}^{2}, \ldots, s_{0}^{j}, \ldots\right\}$ that lead to the set of trajectories $\left\{s^{1}, \ldots, s^{j}, \ldots\right\}$ that remain in the scattering region for at least $N$ crossing times the surface of section. From these trajectories $s^{j}$, we select the one that comes closest to the set $I_{Q}$. Let us say that this trajectory is $s^{n}$ $=\left\{s_{0}^{n}, s_{1}^{n}, \ldots, s_{m}^{n}, \ldots s_{N_{c}}^{n}\right\}$, where $N_{c} \geqslant N$ denotes the number of times that this selected trajectory passes through the surface of section, and $\left(s_{d}^{n}\right)$ for $d \leqslant N_{c}$ is the point of this trajectory in the surface of section that comes closest to the set $I_{Q}$ by say its point $\left(r_{e}^{m}\right)$. If we take this selected trajectory and obtain its time inverse, we have what we shall call the reference trajectory. That is, a trajectory that passes near the point $\left(r_{c}^{m}\right)$ of the trajectory $r^{m}$ and after $N_{c}$ subsequent crossings of the surface of section, it comes near the point $P$. (Recall that to get the points of the time inverse trajectory in the surface of section we replace each $t_{i}$ by $-t_{i}$, and reverse the time sequence of the points.)

The general situation of two orbits that come close to one another is depicted in Fig. 4, where an arbitrary surface of section was defined. There we can see the trajectories $X$ and $Y$, which have the points $X_{j}$ and $Y_{k}$ as the respective position where the orbits come closest to each other.

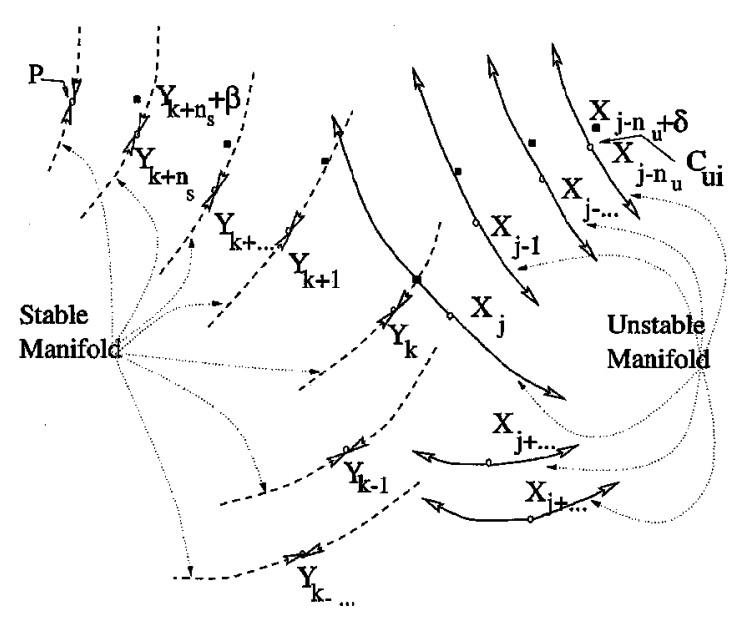

FIG. 5. Targeting method, solving for $\alpha$ and $\beta$ the following equation: $f^{n_{u}}\left(X_{j-n_{u}}+\delta e_{\delta}\right)=f^{-n_{s}}\left(Y_{k+n_{s}}+\beta e_{\beta}\right)$.

For a hyperbolic situation, associated to each point on the invariant set there are stable and unstable manifolds. We introduce at the position $Y_{k+n_{s}}$ a small perturbation $\dot{\beta} e_{\beta}$ where $e_{\beta}$ is a unit vector in the direction of the perturbation (see Fig. 5), and iterate this perturbed point $n_{s}$ times backward in time. This will typically generate a nearby trajectory that will deviate progressively from the original trajectory at each backward iteration, expanding away from $Y$ along the direction of the stable manifold at the points on the orbit $Y$ $[25,26]$. (We assume that the direction of the small perturbation $\hat{\beta} e_{\beta}$ is not precisely such that it has no component in the stable direction.) We also introduce a small perturbation $\hat{\delta} e_{\delta}$ to the orbit $X$ at the iteration $j-n_{u}$ where $e_{\delta}$ is a unit vector in the direction of the perturbation (see Fig. 5), and iterate this perturbed point forward in time $n_{u}$ iterates. This will typically generate a nearby trajectory that will deviate progressively from the original trajectory at each forward iteration, expanding away from $X$ along the direction of the unstable manifold at the points on the orbit $X[25,26]$. Consider that we can find values of the small perturbations $\hat{\delta}$ and $\hat{\beta}$ that solve the equation

$$
f^{n_{u}}\left(X_{j-n_{u}}+\hat{\delta} e_{\delta}\right)=f^{-n_{s}}\left(Y_{k+n_{s}}+\hat{\beta} e_{\beta}\right)
$$

This means that we have found a shadow trajectory that at time $j-n_{u}$ has a point that is $\hat{\delta}$ away from $X_{j-n_{u}}$ and at $n_{s}$ $+n_{u}$ forward iterations in time is a $\hat{\beta}$ distance from the point $Y_{k+n_{s}}$, of the trajectory $Y$. Thus the numbers $n_{u}$ and $n_{s}$ must satisfy $n_{s} \leqslant N_{c}-k$ and $n_{u}<j$. (Note that, since $e_{\beta}$ is not necessarily aligned with the stable manifold at $Y_{k+n_{s}}$, forward iterates of $Y_{k+n_{s}}+\hat{\beta} e_{\beta}$ (if they exist) are expected to diverge from the trajectory $Y$ ). Note that Eq. (8) can be solved for $\delta$ and $\beta$ by the Newton-secant method.

This method can be straightforwardly applied to the previously discussed situation. The trajectory $X$ is associated to the trajectory that passes through the point $\left(r_{0}^{m}\right)$, which is in the vicinity of $Q$, while the trajectory $Y$ is associated to the reference trajectory that passes through the point $\left(s_{d}^{n}\right)$. Note 
that starting with $Q$, two perturbations are necessary in order to obtain the solution trajectory, which is the trajectory that connect the neighborhood of $Q$ to the neighborhood of $P$. Thus, the first perturbation is to put the trajectory in the point $r_{0}^{m}$, while the other is the $\hat{\delta}$ gotten by using the previously discussed method. This last perturbation will yield an orbit that approaches the reference trajectory by following the direction of the stable manifold at the points of the reference trajectory, and thus comes close to the point $P$.

As this approach is a generalization of the one that appears in Ref. [12], it inherits the qualities of the original approach. Thus, our approach works very well, is robust enough to deal with nonideal effects, and can be used as a targeting procedure between any two points inside the chaotic scattering region. In particular, this is the situation that we deal with in the case of the Hill's problem. Here, our goal is, starting from a given value of $h$, to end up in a situation of capture, where $M_{2}$ and $M_{3}$ are kept evolving in relative motion of periodic orbit. In order to accomplish this, the target method is used to guide the trajectory to the neighborhood of the desired orbit, while the previously described control of chaos method keeps the trajectory on the periodic orbit. The details of this approach is presented in the Sec. IV.

\section{HILL'S EQUATIONS}

Let us assume that the mass of either satellite is small compared to the mass of the planet, i.e.,

$$
m_{1} \gg m_{2} \text { and } m_{1} \gg m_{3} \text {, }
$$

where $m_{i}$ is the mass of body $M_{i}$. The ratio of the two masses $m_{2}$ and $m_{3}$ can be arbitrary, but fixed. Thus, we define

$$
m=m_{1}+m_{2}+m_{3}
$$

and

$$
\mu=\frac{m_{2}+m_{3}}{m_{1}} .
$$

Let us assume that the distance between the two satellites is small compared to their distance to the planet. Thus, the two satellites can be approximately considered as a single body in orbit around the planet. We call this orbit as the mean orbit [27-29], and we assume it is a circular orbit with radius $a_{0}$. The angular velocity of $m_{2}$ and $m_{3}$ on this mean orbit is

$$
\omega_{0}=\sqrt{G m a_{0}^{-3}} .
$$

Let $X_{i}, Y_{i}$ be the coordinates of body $i$ in an inertial system. The equations of motion in this reference system are as follows:

$$
\ddot{X}_{1}=\frac{G m_{2}\left(X_{2}-X_{1}\right)}{R_{12}^{3}}+\frac{G m_{3}\left(X_{3}-X_{1}\right)}{R_{13}^{3}},
$$

$$
\ddot{Y}_{1}=\frac{G_{m_{2}}\left(Y_{2}-Y_{1}\right)}{R_{12}^{3}}+\frac{G m_{3}\left(Y_{3}-Y_{1}\right)}{R_{13}^{1}},
$$

and similar equations for $\ddot{X}_{2}, \ddot{Y}_{2}, \ddot{X}_{3}$, and $\ddot{Y}_{3}$, where $G$ is the gravitational constant, and $R_{i j}$ is the distance between the bodies $i$ and $j$, i.e.,

$$
R_{i j}=\sqrt{\left(X_{i}-X_{j}\right)^{2}+\left(Y_{i}-Y_{j}\right)^{2}} .
$$

We introduce dimensionless coordinates by

$$
X_{i}^{\prime}=\frac{X_{i}}{a_{0}}, \quad Y_{i}^{\prime}=\frac{Y_{i}}{a_{0}}, \quad m_{i}^{\prime}=\frac{m_{i}}{m}, \quad t^{\prime}=\omega_{0} t,
$$

so that in these new variables, the gravitational constant, the angular velocity, the total mass of the system, and the radius of the mean orbit are all equal to 1. In Refs. [27-29] it is shown that when the satellites are moving in the neighborhood of each other and the movement is described using synodic coordinates, i.e., in a coordinate system that rotates with angular velocity $\omega_{0}$, a sequence of transformation can be applied, resulting in the following equations for the relative coplanar motion of the satellites described in $\xi$ and $\eta$ coordinates, which are called Hill's equations,

$$
\dot{\xi}=u, \quad \dot{\eta}=v, \quad \dot{u}=2 v+3 \xi-\frac{\xi}{\rho^{3}}, \quad \dot{v}=-2 u-\frac{\eta}{\rho^{3}} .
$$

Hill's equations admit the integral

$$
\Gamma=3 \xi^{2}+\frac{2}{\rho}-u^{2}-v^{2},
$$

which is known as Jacobi integral by analogy with the restrict problem.

We assume that the two satellites are on circular orbits before their encounter, with radii $a_{2}$ and $a_{3}$, both of them close to $a_{0}$. Thus, we can write

$$
\begin{aligned}
& a_{2}=a_{0}\left(1+\mu^{1 / 3} h_{2}\right), \\
& a_{3}=a_{0}\left(1+\mu^{1 / 3} h_{3}\right) .
\end{aligned}
$$

These circular orbits appear in the system of coordinates that we are using as follows:

$$
\xi=h, \quad \eta=-\frac{3}{2} h(t-\tau),
$$

where $h=h_{3}-h_{2}$. In this equation $\tau$ is a trivial parameter that can be eliminated by a change of the origin of time. Furthermore, the Jacobi integral [Eq. (17)] can be written in terms of the $h$ as

$$
\Gamma=\frac{3}{4} h^{2} .
$$

Therefore, our problem depends on just the parameter $h$, which is called the impact parameter. We can say that the orbits which we are considering form a one-parameter family. 

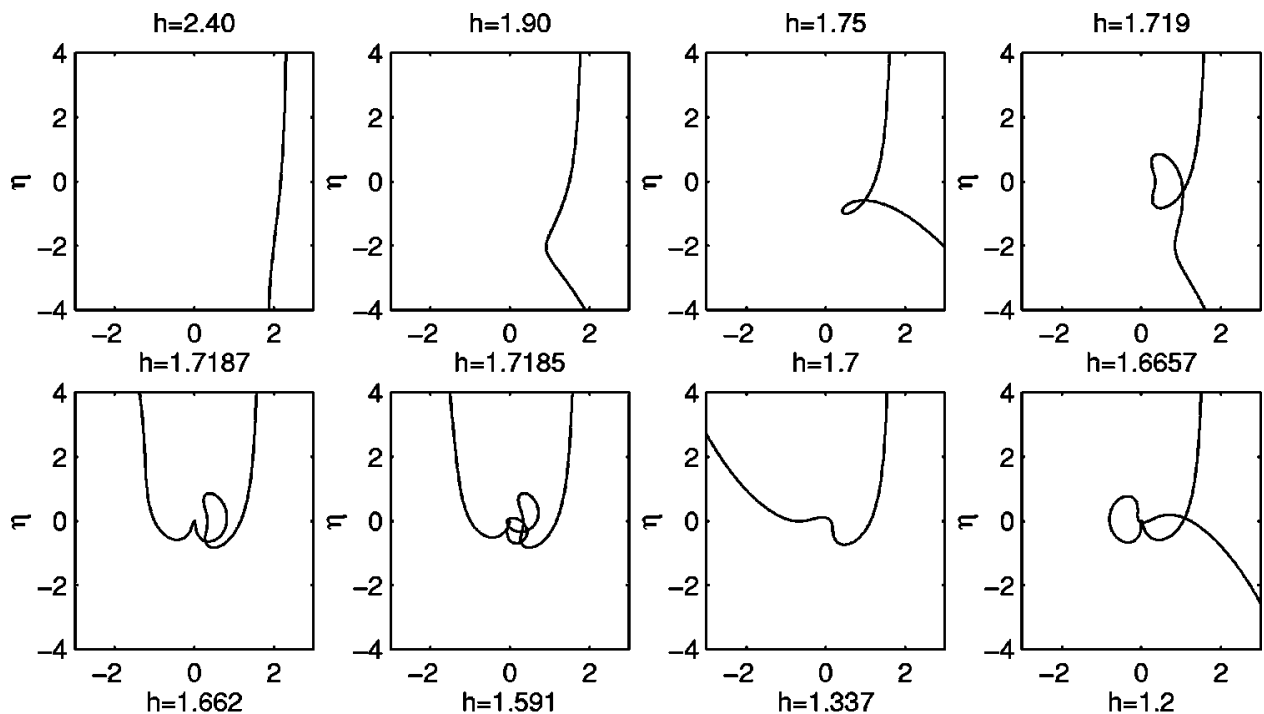

FIG. 6. Trajectories describing the relative motion of the two small bodies for different values of the impact parameter $h$. The variables are dimensionless.
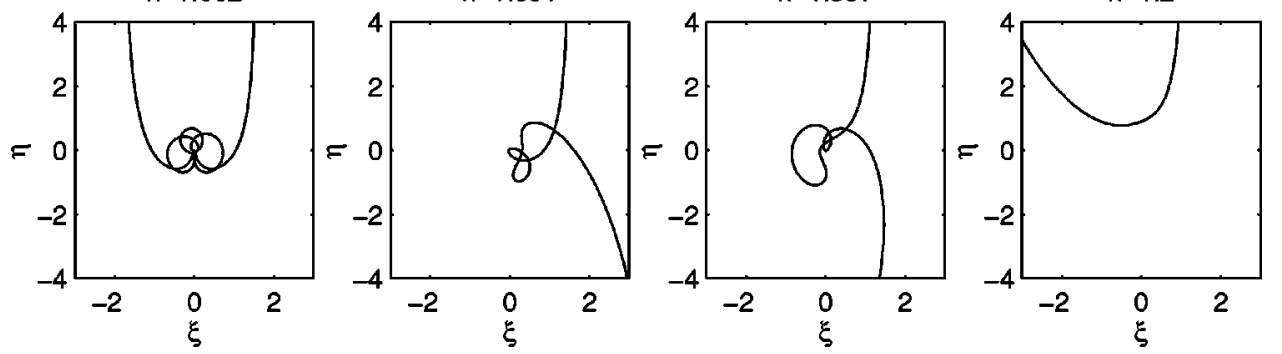

After the encounter, when the satellites are sufficiently far apart, their mutual attraction becomes negligible, and each satellite describes a Keplerian orbit around the planet. However, these orbits are no longer circular. In the system of coordinates that we are using, the asymptotic form of the motion is the following:

$$
\begin{gathered}
\xi=h^{\prime}+k^{\prime} \cos (t-\varphi) \\
\eta=-\frac{3}{2} h^{\prime}(t-\varphi)-\frac{4}{3} s h^{\prime-2} \ln \left[-\frac{3}{2} h^{\prime}(t-\tau)\right]-2 k^{\prime} \sin (t-\varphi)
\end{gathered}
$$

with

$$
s=\operatorname{sgn}(\eta)
$$

In this equation, $h^{\prime}$ is called the impact parameter, as before, while $k^{\prime}$ is the reduced eccentricity. This parameter is related to the actual eccentricities $e_{2}^{\prime}$ and $e_{3}^{\prime}$ of $M_{2}$ and $M_{3}$ by

$$
k^{\prime}=\mu^{-1 / 3} \sqrt{e_{2}^{\prime 2}+e_{3}^{\prime 2}-2 e_{2}^{\prime} e_{3}^{\prime} \cos \gamma^{\prime}},
$$

where $\gamma^{\prime}$ is the angle between the semimajor axes.

Let us now analyze the family's behavior as the parameter changes. To understand the motion of the satellites, we can think about the special case where the mass of one of them, say $m_{2}$ is much larger than the other one, i.e., $m_{2} \gg m_{3}$. For this case, the origin of the relative coordinate system is on $M_{2}$ and the curves just represent the motion of $M_{3}$.

The problem that we are considering is typically characterized by having three well-defined phases. In the first phase, the satellites approach from each other; in the second phase, the two satellites remain close to each other and a complex relative motion takes place; in the last phase, the two satellites move away from each other. Thus, this problem is a typical case of a classical scattering.

For large values of the parameter $h$, the orbit of the lightest satellite is only slightly perturbed, as can be seen on Fig. 6. As the parameter diminishes, the perturbation increases and a loop appears. After that, the shape of the orbit changes rapid with the parameter. Continuing to decrease the value of the parameter, we find an interval in which the shape of the orbits changes wildly. Inside this interval, there is an extremely high sensitivity of the shape of the orbit to just small changes in the parameter. After this interval, as the parameter is reduced, the shape of the orbit changes continuously and slowly with the parameter. Then a new interval of rapidly changes of the shape of the orbit with the variation of the parameter appears, after which follows another interval of wild variation. This succession of behaviors continues to happen as the parameter is diminished, until the appearance of horseshoe orbits.

Figure 7 represents the scattering function, which is the final impact parameter $h^{\prime}$ as a function of the initial impact parameter $h$ for this problem. We observe intervals in which the dependence of $h^{\prime}$ with $h$ is poorly resolved. In fact, for these intervals, the situation of poorly resolved dependency of $h^{\prime}$ to variations in $h$ persists, no matter how we improve the resolution of the scale. Thus, even high magnification of these unresolved intervals does not reveal a smooth curve. This phenomenon is called chaotic scattering.

Chaotic scattering is characterized by "sensitive dependence" of output variables that characterize the particle trajectory after the scattering to small changes in an input vari- 

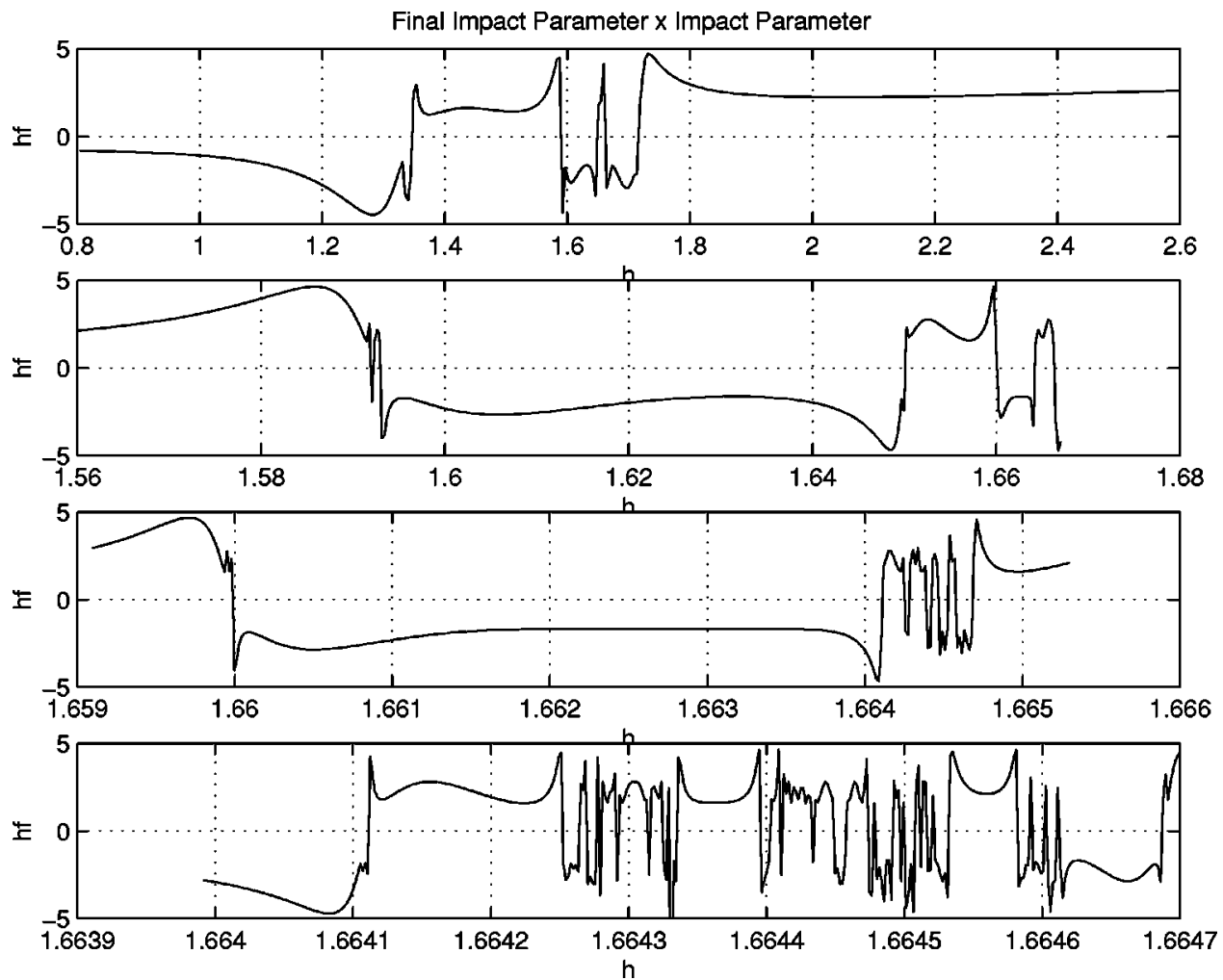

FIG. 7. Scattering function for the interaction of the satellites, which is the final impact parameter $h^{\prime}$ as a function of the initial impact parameter $h$. There are intervals in which the dependence of $h^{\prime}$ with $h$ is poorly resolved even under improvement of the resolution scale. The parameters are dimensionless. able that characterizes the trajectory before scattering [10]. This phenomenon has received much attention because many fundamental physical situations are of this type [27,1,3032]. We say that the scattering function (i.e., the output as a function of the input) is singular at a particular value of the input variable if any interval, containing that input value, produces output variable values in a nonzero range that does not approach zero as the size of the input interval approaches zero. Thus two inputs that are arbitrarily close to a singular value can produce very different outputs. When the set of singular input variables is uncountable, and occurs on a cantor set, we call the situation chaotic scattering, and we say that there is sensitive dependence of the output to small changes in the input.

The dynamics of scattering in chaotic situation can be explained by the existence of a saddle chaotic invariant set [24], formed by the intersection of its stable and unstable manifolds, where the stable and unstable manifolds each consist of a cantor set of roughly parallel surfaces. When a particle enters a scattering region close to the stable mani-
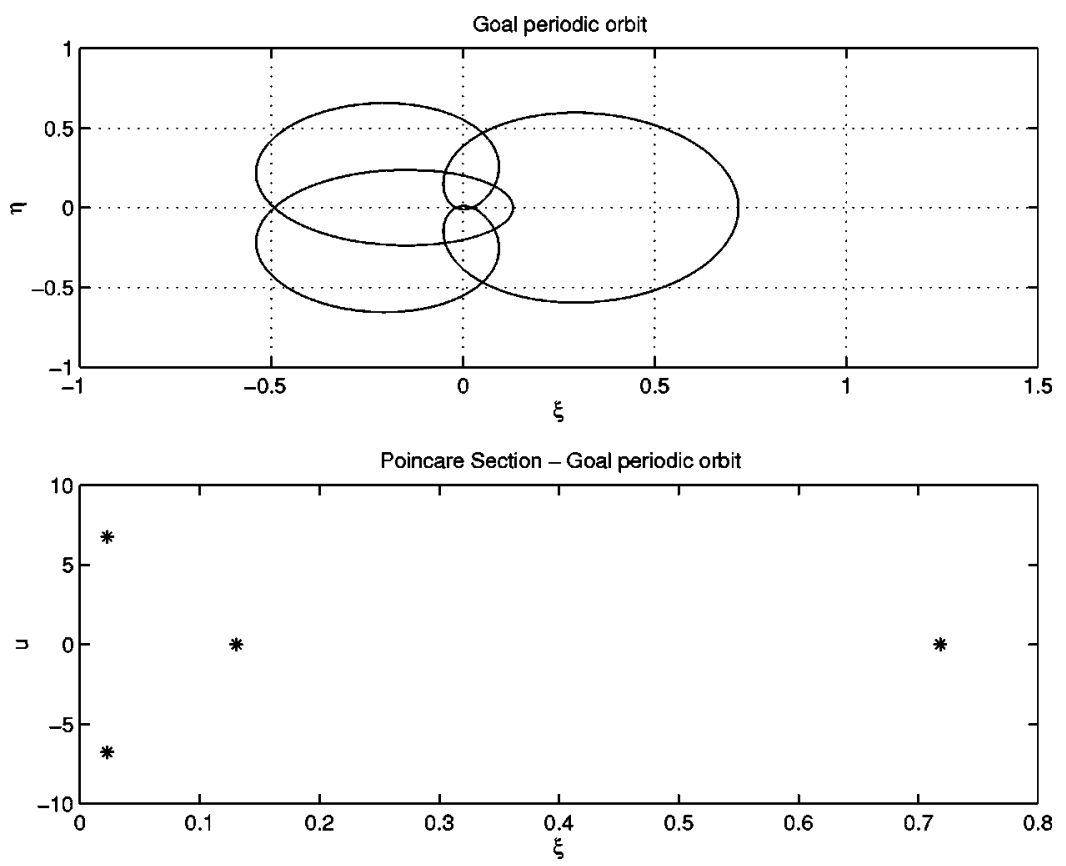

FIG. 8. The desired unstable periodic orbit to be used for stabilizing the relative motion of the two small bodies $M_{2}$ and $M_{3}$. The variables are dimensionless. 

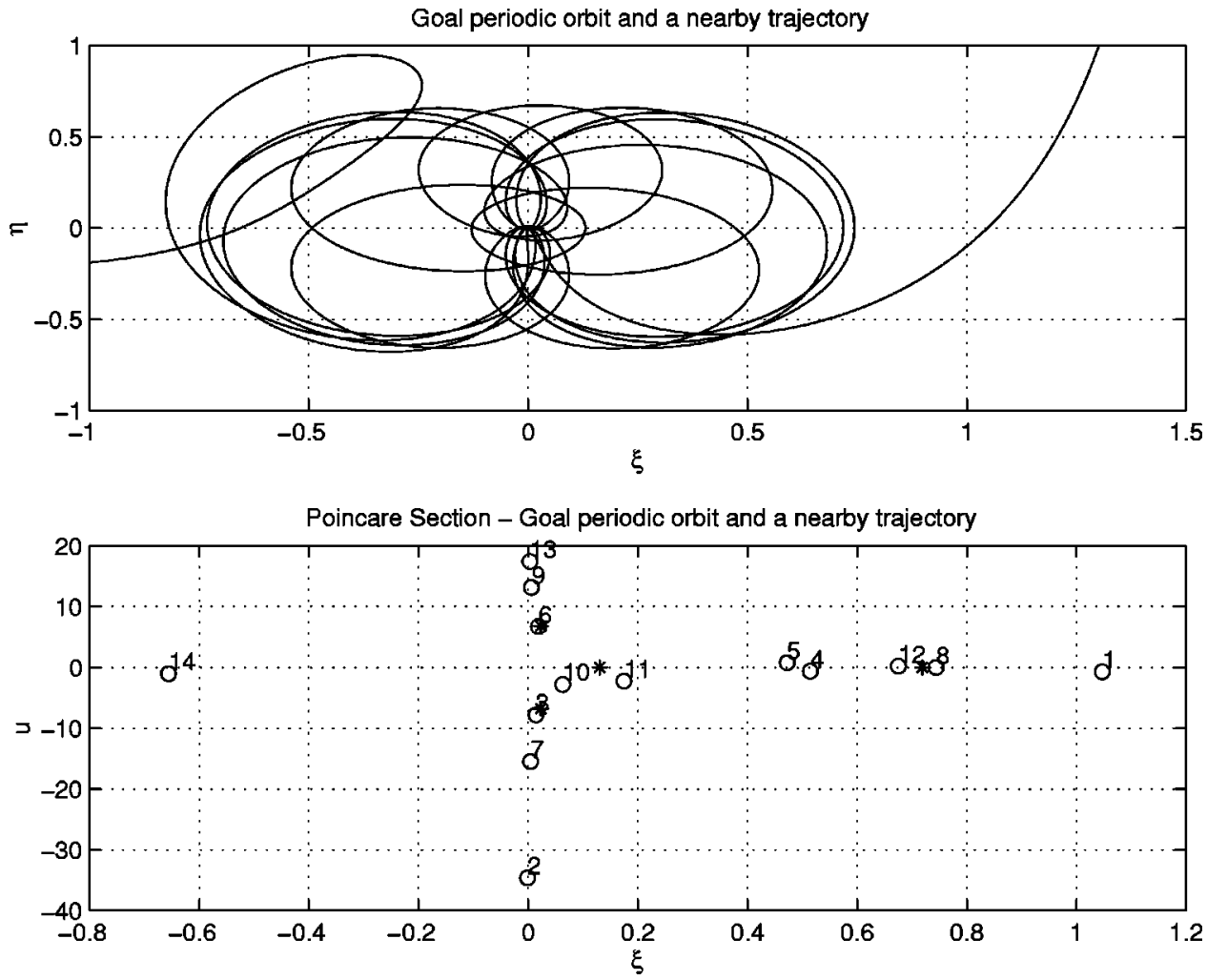

FIG. 9. The desired unstable periodic orbit and a trajectory found in the neighborhood of the impact parameter $h_{0}$ that come very close to the unstable periodic orbit. In the Poincare section, the unstable periodic orbit is represented by ${ }^{*}$, while the nearby trajectory is represented by $\bigcirc$. The numbers identify the order in which the trajectory crosses the Poincaré section. The variables are dimensionless. fold, it stays near the saddle chaotic set for some time, and then escapes following a path close to the unstable manifold. The closer it initially is to the stable manifold, the more time it spends in the scattering region. If the initial condition of the particle is precisely on the stable manifold, the particle stays in the scattering region forever, and small deviations from this situation can lead to wild variations of the output [10,24,32-36].

Embedded in this saddle chaotic invariant set is a numerable and infinite set of unstable periodic orbits. These orbits are exploited in Sec. V to keep the satellites in orbit of one another by using chaos control strategy.
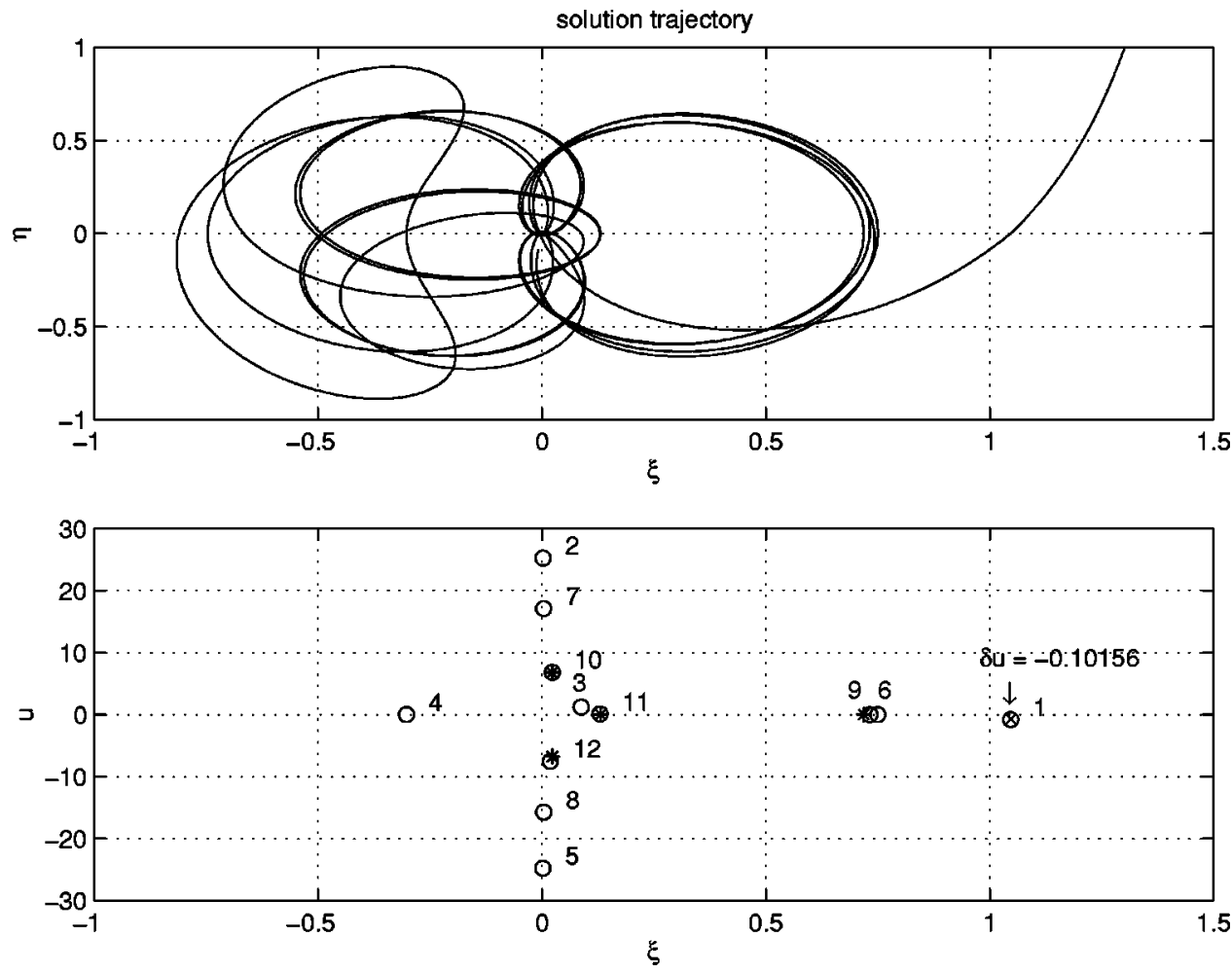

FIG. 10. The solution trajectory that results from the use of the targeting method. The perturbation to the nearby trajectory is applied in the first point in which the trajectory crosses the Poincare section, as indicated in the figure. Due to the perturbation, the trajectory is directed to follow the unstable periodic orbit. Points in the Poincaré section that belong to the unstable periodic orbit are represented by $*$. Variables and the parameter are dimensionless. 


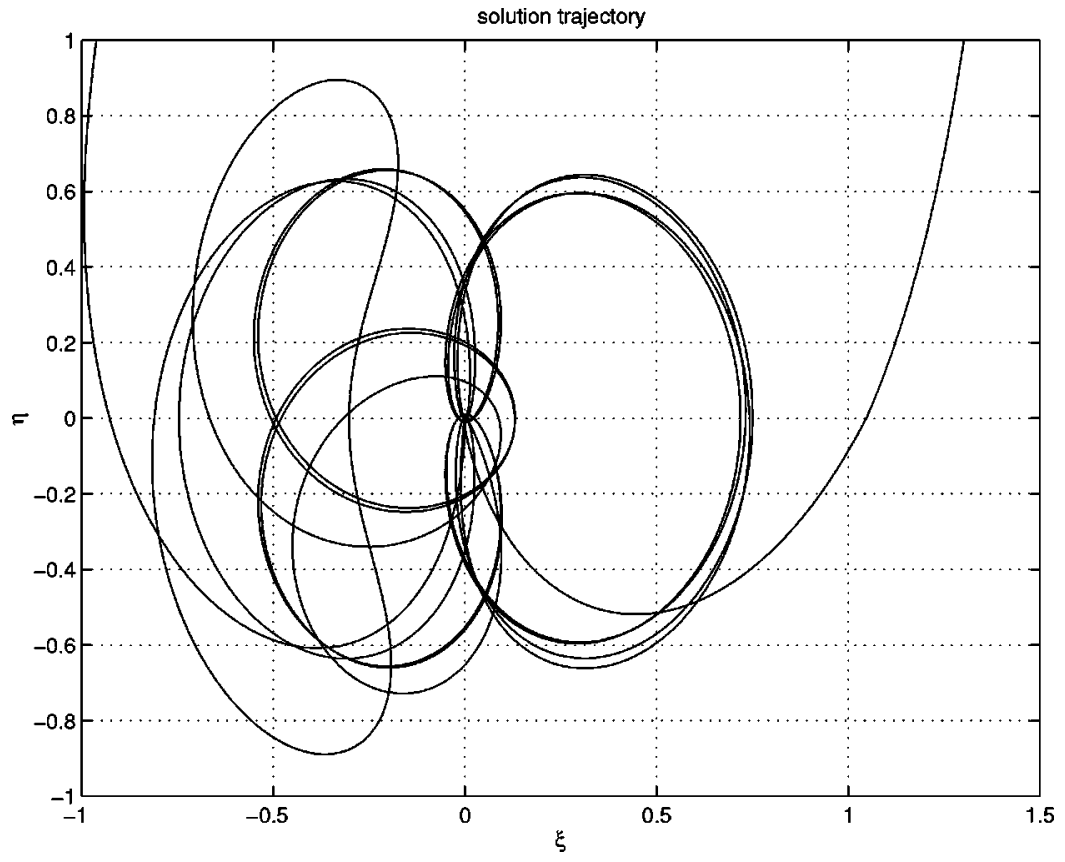

FIG. 11. Without control, the trajectory that results from the application of our targeting method follows for while the unstable periodic orbit and moves away from it by following the unstable manifold of the unstable periodic orbit. It means that one satellite stays in orbit around the other for some time. After that, they move away from each other. Variables are dimensionless.

\section{CONTROLLING A SATELLITE ENCOUNTER}

In this section we apply the previously discussed ideas about control of chaos and targeting in a chaotic scattering to typical scenario where this phenomenon happens. We use the Hill's equation as a model of a satellite encounter. The dynamical behavior of this problem was discussed in Sec. IV. We start by introducing a Poincaré section $\eta=0$, mapping the $(\xi, u)$ plane to itself, whenever the trajectory traverses the Poincaré section with $v<0$. Thus, the Poincare return map for fixed $\Gamma$ defines a two-dimensional map.

To apply the discussed targeting and control of chaos methods, starting from a given value of $h_{0}$, which in this problem performs the role of $Q$ for the above target method, we identify in the Poincare section an unstable periodic orbit that is nearby the original orbit for $h_{0}$ and imply an orbital motion for the satellites that fulfill the mission specifications. The points of this orbit are used as the targeting for our targeting strategy. Thus, the first step is to identify a set of unstable periodic orbits and from this set select one that will be used to stabilize the motion of the satellites. Hereafter we call this unstable periodic orbit as the parked orbit. We are assuming that for the given $h_{0}$ the Hill's problem presents a chaotic scattering behavior, otherwise our methods do not work.

Periodic orbits are found for values of the impact parameter for which the chaotic scattering is present using Schmelcher-Diakonos method [37], in association with the
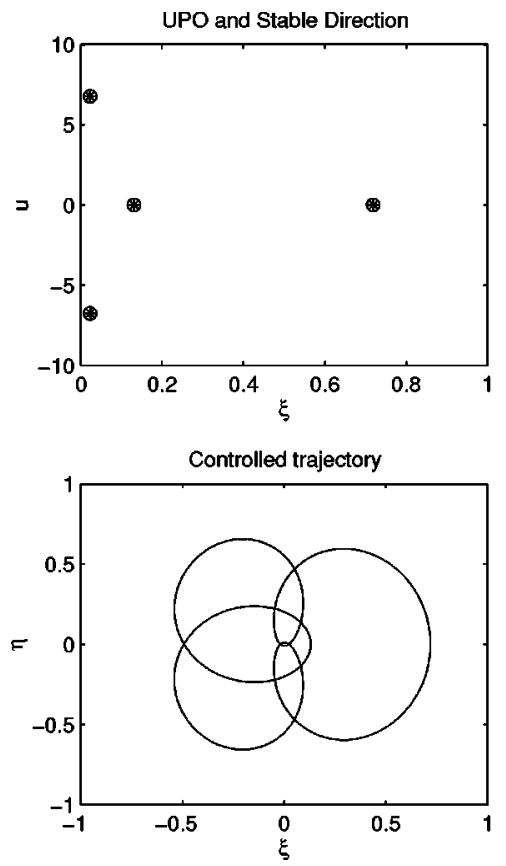
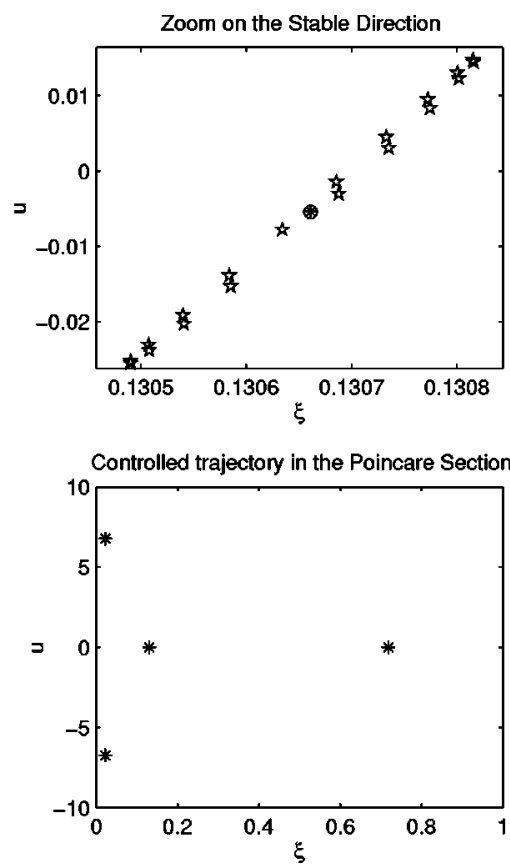

FIG. 12. Using chaos control strategy, the trajectory keeps following the unstable direct orbit, which means that one satellite stays in orbit around the other. In the figure, we show the results from the application of the chaos controls strategy. Variables are dimensionless. 


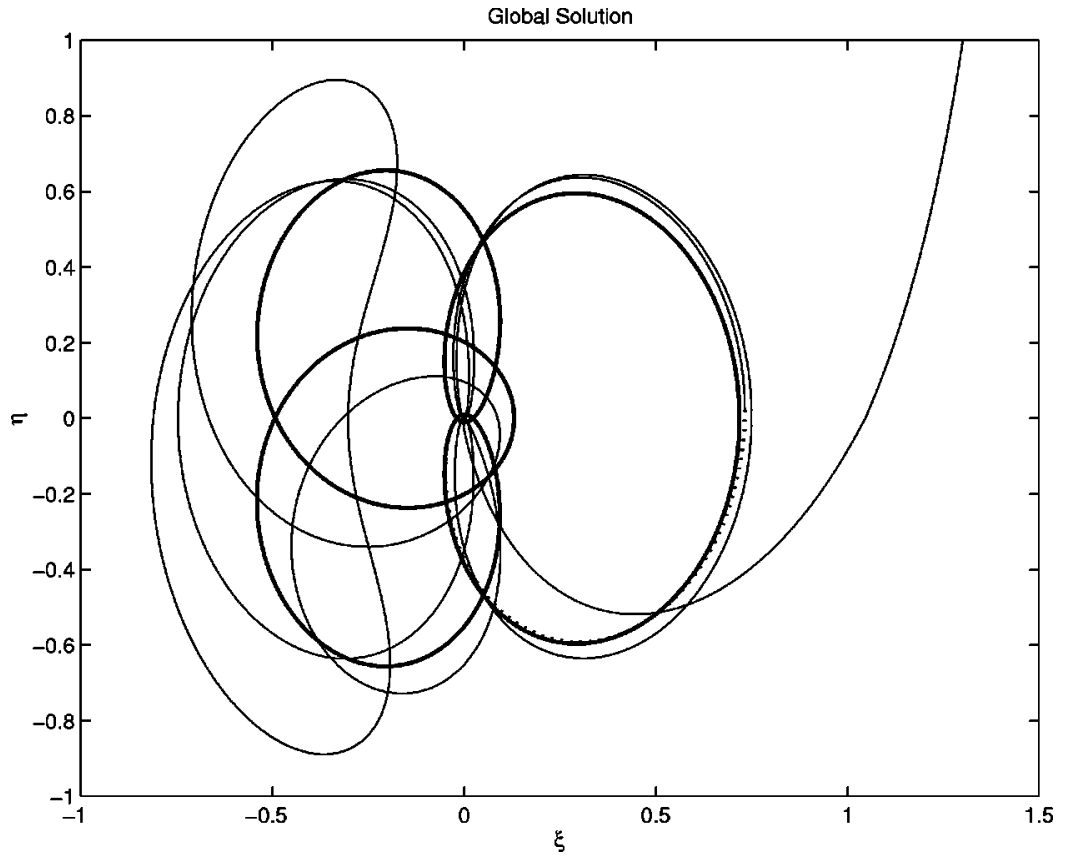

FIG. 13. The final trajectory that results from the application of both the targeting and the chaos control strategy. The part of the trajectory that evolves under the action of the control strategy appears in dotted line. Variables are dimensionless.
Newton-Raphson method (NR). The first method is applied to each point of a set of starting points defined by a uniform grid of initial conditions over the state space. For each point of this set, the method converges to a solution. For distinct starting points the method may converge to the same solution, which is particularly true if the number of the points of the grid is larger than the number of unstable periodic orbits embedded in the chaotic invariant set. Furthermore, this process of scanning the state space by using the points of the grid must be repeated for each value of period of periodic orbits that we are interested in finding out. From the application of this method, we get the solution points of the desired periodic orbits and also points of quasiperiodic orbits. All these points were then used as the starting points for the Newton-Raphson method. This method not only allows a refinement of the points that belong to periodic orbits, but also discharges eventual points that belong to quasiperiodic orbits.

From the set of unstable periodic orbits, we chose the one that best fits our purpose concerning the spacecraft mission. To show how the methods of targeting and control of chaos can be used in association, we selected the periodic orbit that appears in Fig. 8. We call this orbit as the parked orbit, as stated before.

After the determination of the parked orbit, the next step is to find a nearby trajectory from $Q$ that come close to the parked orbit. So, introducing small random perturbations to $h_{0}$, we find the perturbation $d h_{r}$ to $h_{0}$ that implies in the orbit of the set that come closest to the parked orbit. This trajectory, which appears in Fig. 9, plays the role of the reference trajectory described in the method. In this figure, the parked orbit is represented in the Poincare section by asterisk, while the reference trajectory is represented by circles with numbers that represent the order in which the trajectory crosses the Poincaré section. Note that the reference trajec- tory come closest to the parked orbit in its sixth crossing of the Poincare section. This point of closest approach $\left(\xi_{c}, u_{c}\right)$ is then used to determine by using Eq. (8) the perturbation $d u_{c}$ to be applied to $\left(\xi_{c}, u_{c}\right)$ so that the perturbed trajectory comes to the neighborhood of the parked orbit. In Fig. 10 we show the problem solution for this specific $h_{0}$. Note that using our targeting method we can end up in a neighborhood of the parked orbit by using just two perturbations.

As the trajectory is in the neighborhood of the parked orbit, it follows it for a while and escapes from it by following the unstable manifold of the parked orbit. It means that one satellite stays in orbit around the other for some time after which they move away from each other. This situation can be seen in Fig. 11.

The satellites can be kept in the parked orbit by using our chaos control strategy. The result of applying our stabilization strategy appears in Fig. 12. In that figure the stable direction found by using our method is presented as well as the controlled trajectory. The global solution, that results from the use of both strategies of targeting and control appears in Fig. 13.

\section{CONCLUSION}

This work presents two main contributions. First, we generalize a previously proposed method for targeting in chaotic scattering so that it can now be used to find a transfer trajectory between any two points located inside the scattering region. Second, we show for the first time that control of chaos and targeting methods can be combined to generate a powerful instrument to drive orbits around a chaotic invariant set even if this set is a nonattractive one. To illustrate how this can be applied, we use a case of practical interest in celestial mechanics in which it is desired to control the evo- 
lution of two satellites that evolve around a large central body. Of course, as our approach is general, it can be used to any situation where chaotic scattering happens. Thus, it can be applied to many fundamental physical situations of chaotic scattering in molecular dynamics, fluid dynamics, mesoscopic systems, chemical reactions, electrodynamics, and nuclear physics.

\section{ACKNOWLEDGMENTS}

This work was supported by CNPq (Brazilian Agency for Research and Technological Development) and FAPESP (The State of São Paulo Research Foundation). E.E.N.M. would like to thank Dr. Maisa de Oliveira Terra for helpful discussions.
[1] P. T. Boyd and S. L. W. McMillan, Chaos 3, 507 (1993).

[2] Z. Kovács and L. Wiesenfeld, Phys. Rev. E 51, 5476 (1995).

[3] A. Péntek, Z. Toroczkai, T. Tél, C. Grebogi, and J. Yorke, Phys. Rev. E 51, 4076 (1995).

[4] Z. Toroczkai, G. Károlyi, A. Péntek, T. Tél, and C. Grebogi, Phys. Rev. Lett. 80, 500 (1998).

[5] R. A. Jalabert, H. U. Baranger, and A. D. Stone, Phys. Rev. Lett. 65, 2442 (1990).

[6] C. C. Rankin and W. H. Miller, J. Chem. Phys. 55, 3150 (1971).

[7] L. Gottdiener, Mol. Phys. 29, 1309 (1975).

[8] A. A. Chernikov and G. Schmidt, Chaos 3, 525 (1993).

[9] K. Richter and D. Wintgen, J. Phys. B 23, L197 (1990).

[10] E. Ott and T. Tél, Chaos 3, 417 (1993).

[11] Y.-C. Lai, M. Ding, and C. Grebogi, Phys. Rev. E 47, 86 (1993).

[12] E. E. N. Macau, Phys. Rev. E 57, 5337 (1998).

[13] G. W. Hill, Am. J. Math. 1, 5 (1878).

[14] P. Goldreich and S. Tremaine, Nature (London) 277, 97 (1979).

[15] P. Goldreich and S. Tremaine, Astrophys. J. 241, 425 (1980).

[16] M. Henon, Nature (London) 293, 33 (1981).

[17] S. F. Dermontt and C. D. Murray, Icarus 48, 1 (1981).

[18] N. Schofield, Mon. Not. R. Astron. Soc. 197, 1031 (1981).

[19] S. H. Dole, Planet. Space Sci. 9, 541 (1962).
[20] E. Ott, C. Grebogi, and J. A. Yorke, Phys. Rev. Lett. 64, 1196 (1990).

[21] C. Grebogi and Y. C. Lai, Syst. Control Lett. 31, 307 (1997).

[22] Y.-C. Lai, T. Tél, and C. Grebogi, Phys. Rev. E 48, 709 (1993).

[23] T. Shinbrot, E. Ott, C. Grebogi, and J. A. Yorke, Phys. Rev. Lett. 65, 3215 (1990).

[24] G.-H. Hsu, E. Ott, and C. Grebogi, Phys. Lett. A 127, 199 (1988).

[25] E. M. Bollt and J. D. Meiss, Physica D 81, 280 (1995).

[26] E. M. Bollt and J. D. Meiss, Phys. Lett. A 204, 373 (1996).

[27] J.-M. Petit and M. Henon, Icarus 66, 536 (1986).

[28] M. Henon and J.-M. Petit, Celest. Mech. 38, 67 (1986).

[29] E. E. N. Macau, IAF-99-A, 106, 1999 (unpublished).

[30] S. Bleher, C. Grebogi, E. Ott, and R. Brown, Phys. Rev. A 38, 930 (1988).

[31] J. Chen, J. Geophys. Res. 97, 15011 (1992).

[32] P. Gaspard and S. A. Rice, J. Chem. Phys. 90, 2225 (1988).

[33] S. Bleher, C. Grebogi, and E. Ott, Physica D 46, 87 (1990).

[34] M. Ding, C. Grebogi, E. Ott, and J. A. Yorke, Phys. Rev. A 42, 7025 (1990).

[35] M. Ding, C. Grebogi, E. Ott, and J. A. Yorke, Phys. Lett. A 153, 21 (1991).

[36] B. Eckhardt, J. Phys. A 20, 5971 (1987).

[37] P. Schmelcher and F. K. Diakonos, Phys. Rev. E 57, 2739 (1998). 